\title{
TEM analyses of in situ presolar grains in pristine matrix material of ordinary chondrite Semarkona
}

\author{
Sheryl Singerling ${ }^{1}$, Larry Nittler ${ }^{2}$, Elena Dobrica ${ }^{3}$, Adrian Brearley ${ }^{4}$ and Rhonda Stroud ${ }^{1}$ \\ ${ }^{1}$ U.S. Naval Research Laboratory, United States, ${ }^{2}$ Carnegie Institution of Washington, United States, \\ ${ }^{3}$ University of Hawai'i at Manoa, United States, ${ }^{4}$ University of New Mexico, United States
}

Presolar grains are dust that predates the formation of the solar system by up to several billion years and include phases such as diamond, silicon carbide (SiC), graphite, oxides, and silicates [1,2]. These grains are identified by their anomalous isotopic compositions, which are the product of formation in circumstellar environments ranging from the atmospheres of evolved low mass stars (AGB stars) to the ejecta produced during the explosive deaths of massive stars (supernovae). Presolar grains are components of the original building blocks of our Solar System, and are preserved to varying degrees in primitive meteorites and interplanetary dust particles (IDPs).

The LL3.0 ordinary chondrite Semarkona is a highly primitive meteorite that escaped significant heating but exhibits pervasive effects from interaction with fluids [3-6]. Recently, amorphous silicate-rich regions of pristine matrix material, which escaped such alteration, have been identified [7]. A NanoSIMS search of these regions identified abundant presolar grains, consistent with minimal processing. By studying presolar grains within the meteorite host (in situ), we can better trace the entire history of an individual presolar grain from its formation in circumstellar environments to its transit through the interstellar medium to its residence in the solar nebula and later on an asteroidal parent body. In situ TEM studies of presolar grains are becoming more common owing to the advent of the NanoSIMS and the FIB liftout technique. Our current study, the first to employ FIB-TEM to investigate the structural and compositional characteristics of in situpresolar grains from an ordinary chondrite, seeks to determine what the properties of the grains can tell us about their histories.

We used TEM to analyze 7 presolar grains - $1 \mathrm{SiC}$ (F2-30a), 1 oxide (F2-37), 2 silicates (F2-9 and F2-30b), and 3 aggregates (F2-23, F2-8, and F1-1). These grains were identified as presolar via automatic NanoSIMS mapping of $\mathrm{C}$ and/or $\mathrm{O}$ isotopes with a $\mathrm{Cs}^{+}$primary ion beam and were remeasured for their $\mathrm{Mg}-\mathrm{Al}$ isotope systematics with an $\mathrm{O}^{-}$beam. An FEI Helios FIB-SEM was used to lay down fiducial markers $(\mathrm{C}$ and $\mathrm{Pt})$ and prepare FIB sections of the grains and surrounding matrix material. A JEOL 2200FS TEM was used to collect BF images, HRTEM images, and SAED patterns, and a Nion UltraSTEM-200X was used to collect HAADF STEM images and EDS maps/spectra, all at $200 \mathrm{kV}$.

The presolar grains are $\mathrm{SiC}$, oxides, silicates, and aggregates that range in size from 120 to $1930 \mathrm{~nm}$ (Table 1) and morphology from euhedral to anhedral. Although some grains are well-crystallized, some silicates are weakly nanocrystalline, showing local areas (10s of $\mathrm{nm}$ ) of crystallinity adjacent to amorphous patches, consistent with previous reports [8 and references therein]. Amorphous materials are adjacent to several presolar grains/aggregates (F2-23, F2-8, and F1-1), and are characterized by compositions more silica-rich than pyroxene $(\mathrm{Ca}+\mathrm{Fe}+\mathrm{Mg} / \mathrm{Si}=0.80-0.82)$. The boundaries between the presolar silicates and the amorphous silicate-rich matrix are difficult to distinguish using textural features. This could imply the boundaries were modified by parent body alteration, consistent with the presence of magnetite rims on Fe-carbides in the pristine matrix [7].

Some grains contain compositional heterogeneities, such as more Al-rich $\mathrm{SiC}$ in regions of high disorder in SiC F2-30a (Fig. 1a-b), Mg-rich and -poor regions in silicate F2-9 (Fig. 1c-d), and Si-rich and Mg-rich regions in the silicate portions of aggregate F1-1. The silicates often show non-stoichiometry (Table 1). Rims were observed on SiC F2-30a (S-rich 10 nm thick) and silicate F2-9 (Mg-rich from 20-40 nm thick) (Fig. 1a-d), and could be the result of parent body alteration. 
The unusually large aggregate F2-8 is distinct from the other presolar grains in terms of its size and phase assemblage (olivine-spinel-pyroxene) (Fig. 2). The olivine is Mg-rich (Fo99), spinel is Al-spinel $\left(\mathrm{MgAl}_{2} \mathrm{O}_{4}\right)$, and pyroxene is $\mathrm{Ca}$ - and $\mathrm{Mg}$-rich $\left(\mathrm{Wo}_{44} \mathrm{En}_{54}\right)$. Evidence for parent body alteration of this assemblage includes amorphous Ca-pyroxene and the presence of Fe,O-rich material within fractures of some components (ol, sp) or at the interfaces between components (ol-sp, px-sp).

All samples show evidence for NanoSIMS beam damage. In SiC F2-30a, the topmost $20 \mathrm{~nm}$ is amorphous (Fig. 1a). In all FIB sections, vesicles were observed near the contact between the meteorite material and the deposited $\mathrm{C}$ or $\mathrm{Pt}$ (Fig. 2b). The vesicles likely stem from $\mathrm{O}^{-}$ion beam damage from NanoSIMS analyses, given that they have not been observed previously from in situ FIB-TEM analyses of presolar grains only analyzed with $\mathrm{Cs}^{+}$ions.

Some insights into the histories of the grains can be inferred from our in situ studies. The presolar silicates which are weakly nanocrystalline, compositionally heterogeneous, and/or non stoichiometric in composition indicate that condensation around their progenitor AGB stars occurred under non-equilibrium conditions [8]. In contrast, the phase assemblage (Mg-rich ol, $\mathrm{Al}-\mathrm{sp}, \mathrm{Ca}-\mathrm{px})$ in aggregate $\mathrm{F} 2-8$ is consistent with equilibrium condensation under a range of conditions appropriate for AGB stars (e.g., $\mathrm{C} / \mathrm{O}<0.96, \mathrm{P}=10^{-3}$ bars, $\mathrm{T}=1265-$ $1380 \mathrm{~K}$ ) [9]. The presence of alteration textures in some grains implies that, even in pristine matrices, the presolar grains experienced parent body alteration.

Table 1. Textural and compositional information on 7 presolar grains studied

\begin{tabular}{|c|c|c|c|}
\hline Grain & Phase(s) & Size (nm) & $\mathrm{Ca}+\mathrm{Fe}+\mathrm{Mg} / \mathrm{Si}$ \\
\hline F2-30a & $\mathrm{SiC}$ & $210 \times 90$ & - \\
\hline $\mathrm{F} 2-37$ & $\mathrm{Sp}$ & $120 \times 80$ & - \\
\hline F2-9 & NS & $290 \times 80$ & 0.38 (Mg-poor), 1.66 (Mg-rich), 2.38 (rim) \\
\hline$F 2-30 b$ & NS & $230 \times 120$ & 0.77 \\
\hline $\mathrm{F} 2-23$ & Ol-Sulfide & $170 \times 150$ & 1.95 \\
\hline F2-8 & Ol-Sp-Cpx & $1930 \times 500$ & $1.91(\mathrm{Ol}), 1.06(\mathrm{Cpx})$ \\
\hline
\end{tabular}


$\mathrm{Sp}$ - spinel $\left(\mathrm{MgAl}_{2} \mathrm{O}_{4}\right), \mathrm{NS}$ - non-stoichiometric silicates, $\mathrm{Ol}$ - olivine $\left((\mathrm{Fe}, \mathrm{Mg})_{2} \mathrm{SiO}_{4}\right), \mathrm{Cpx}-\mathrm{Ca}$-pyroxene $\left((\mathrm{Ca}, \mathrm{Fe}, \mathrm{Mg})_{2} \mathrm{Si}_{2} \mathrm{O}_{6}\right)$

Work supported by NASA EW grant 80HQTR19T0038 to RMS

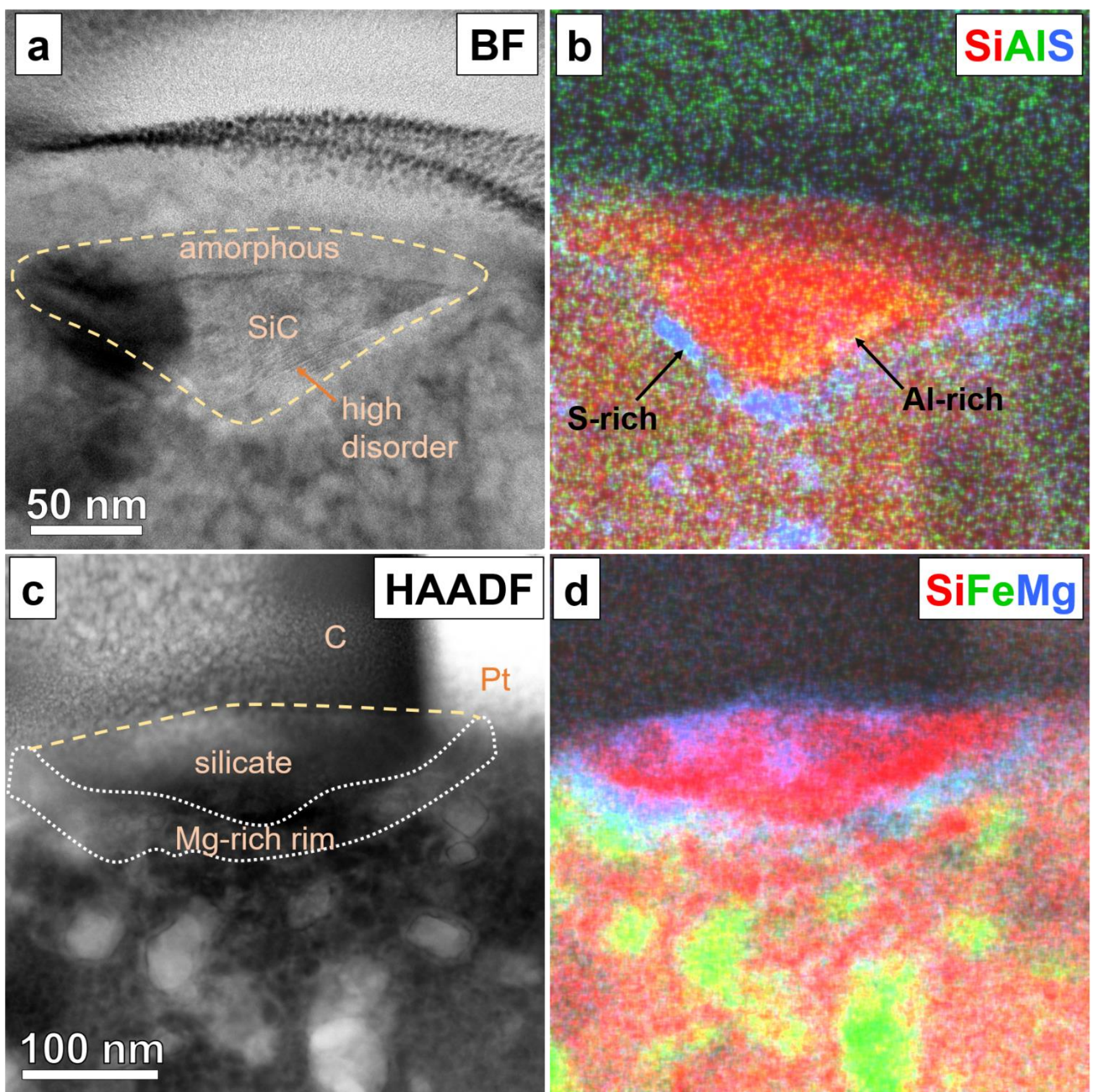

Figure 1. TEM images of presolarSiC F2-30a (a-b) and silicate F2-9 (c-d). The BF image (a) and EDS X-ray map (b) of F2-30a show the amorphous versus crystalline $\mathrm{SiC}$, the Al-rich regions of high-disorder (yellow- 
green), and the S-rich rim (blue). The EDS X-ray map of F2-9 (d) shows Mg-rich (blue) and Mg-poor (red) regions within the silicate as well as a $\mathrm{Mg}$-rich rim (light blue).

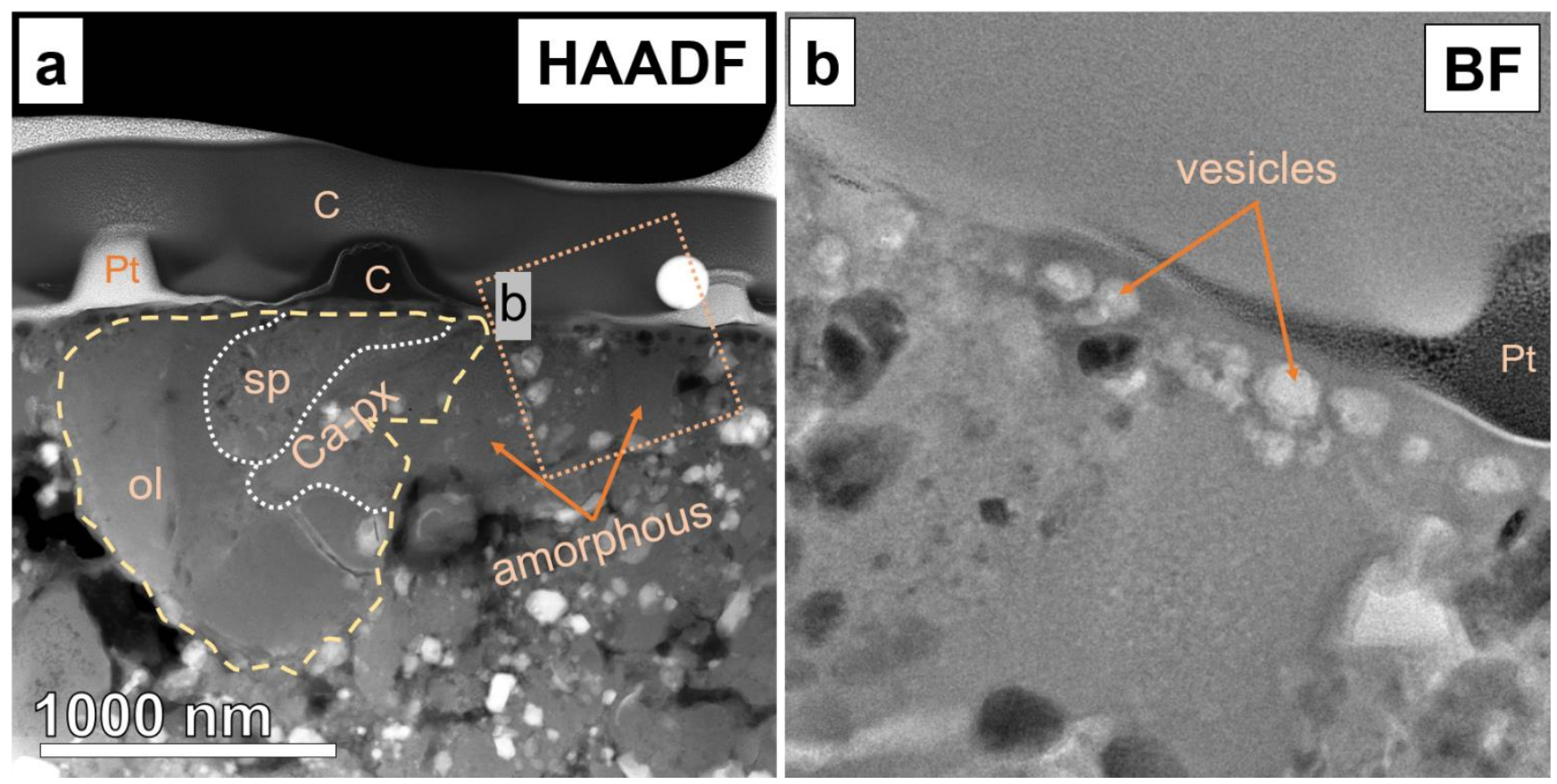

Figure 2. TEM images of presolar aggregate F2-8. The HAADF image of F2-8 (a) shows the presence of distinct regions (ol - olivine, Ca-px - Ca-pyroxene, sp - spinel) within the overall aggregate. A higher magnification BF image (b) shows the vesicles near the surface of the FIB section that likely indicate damage from the O- ion beam used for NanoSIMS analyses.

\section{References}

[1] Heck P. R. et al. PNAS117 (2020) 1884-1889.

[2] Nittler L. R. and Ciesla F. Annu. Rev. Astron. Astrophys. 54 (2016) 53-93.

[3] Hutchison R. et al. GCA 51 (1987) 1875-1882.

[4] Alexander C. M. O’D. et al. GCA 53 (1989) 3045-3057.

[5] Krot A. N. et al. GCA 61 (1997) 219-237.

[6] Grossman J. N. and Brearley A. J. MAPS 40 (2005) 87-122.

[7] Dobrică E. and Brearley A. J. MAPS 55 (2020) 649-668.

[8] Floss C. and Haenecour P. Geochem. J.50 (2016) 3-25.

[9] Ebel D. S. MESSII (2006) 253-277. 